\title{
Aktuelle Versorgungsstrategie der Calcaneusfrakturen
}

\author{
Stefan Rammelt, Johann Marian Gavlik, Sven Barthel, Rene Grass, Hans Zwipp
}

\section{Zusammenfassung}

Das Fersenbein nimmt eine Sonderstellung unter den Fußknochen ein. Durch die spongiöse Binnenstruktur und die Beteiligung an vier Gelenkflächen entstehen am Calcaneus in drei Vierteln der Fälle Frakturen mit Gelenkbeteiligung, in $60 \%$ fünf Hauptfragmente, häufig mit Stauchungsund Trümmerzonen. Die herausragende Funktion des Fersenbeines als hinterer Abschnitt des Fußlängsgewölbes und kräftiger Pfeiler der lateralen Fußsäule wird bei jeglicher Dislokation empfindlich gestört. Die unter hoher Belastung stehende posteriore $\mathrm{Ge}$ lenkfacette, welche für die Beweglichkeit im Rückfuß und damit für den Abrollvorgang entscheidend ist, wird selbst bei geringsten Verwerfungen dauerhaft geschädigt. Das Ziel der Versorgung von Calcaneusfrakturen muss daher die exakte Wiederherstellung der äußeren Form und die subtile Gelenkrekonstruktion sein, was sich in den meisten Fällen aufgrund des Ver- letzungsausmaßes nur mit einer offenen Reposition und übungsstabiler, nicht gelenkübergreifender Plattenosteosynthese erreichen lässt. Die prekäre Weichteilbedeckung macht eine sorgsame Präparation und exakte Planung des Operationszeitpunktes obligat. Bei Beachtung dieser Punkte liegt die Rate guter und sehr guter Behandlungsergebnisse zwischen 65 und $80 \%$. Somit hat das operative Vorgehen mit anatomischer Rekonstruktion und frühfunktioneller Übungsbehandlung zu einer deutlichen Verbesserung der Prognose dieser schweren Verletzungen geführt. Neuere Tendenzen, wie die arthroskopische Gelenkkontrolle in Verbindung mit offener oder perkutaner Reposition, die frühe Lappendeckung bei offenen Frakturen, der Einsatz winkelstabiler Implantate bei zunehmendem Verzicht auf eine autologe Spongiosaplastik und die Arthrolyse im Rahmen der Implantatentfernung sollten perspektivisch eine weitere Verbesserung

\section{Einleitung}

Die komplizierte spongiöse Architektur und spärliche Weichteilbedeckung des Fersenbeines macht die operative Versorgung intraartikulärer Calcaneusfrakturen zu einer der größten Herausforderungen in der Unfallchirurgie. Dies spiegelt sich in einer wechselvollen Geschichte mit zahlreichen Änderungen im generellen Therapiekonzept wider. Bereits im 19.Jahrhundert haben einige Chirurgen noch ohne die Möglichkeit der Röntgendiagnostik einen Traktionsapparat einge-

OP-JOURNAL 2001; 17: 45-54

(C) Georg Thieme Verlag Stuttgart · New York setzt und mit der offenen Einrichtung von Fersenbeinfrakturen begonnen [24]. Goff beschrieb 1936 in einer Übersichtsarbeit bereits 41 verschiedene offene und halboffene Operationsverfahren, welche von 1720 bis zum Anfang des 20. Jahrhunderts propagiert wurden [10].

Obgleich bereits Ende der 20er Jahre von den Pionieren der operativen Fersenbeinbruchbehandlung wie Leriche die primäre Schraubenosteosynthese, von Lenormant und Palmer die essentielle Wiederaufrichtung und laterale Unterfütterung der subtalaren Gelenkfläche empfohlen wurde, dominierten aufgrund der zahlreichen Infektverläufe und wenig zufriedenstellender funktioneller Ergebnisse konservative und semi-operative Verfah- ren lange Zeit das therapeutische Regime [3]. Erst in den 80-er Jahren wurde von einer zunehmenden Anzahl von Autoren eine primär übungsstabile, nicht gelenkübergreifende Osteosynthese favorisiert, wobei erst durch dem routinemäßigen Einsatz des CT eine exakte Frakturanalyse und präoperative Planung ermöglicht wurde $[2,6,18,23]$.

Die Häufigkeit von Calcaneusfrakturen wird in der Literatur mit 1-2\% aller Frakturen angegeben [24], wobei sie mit etwa $60 \%$ die häufigsten Frakturen der Fußwurzel darstellen. Sie gehen in der Mehrzahl mit einer erheblichen Verkürzung, Verbreiterung und einem Achsenknick einher, was die Statik und Dynamik des Fußes im Sinne eines posttraumatischen Knick-Plattfußes erheblich beeinträchtigt. Zudem können bereits kleine Gelenkverwerfungen von 1-2 mm zu schmerzhaften, teilweise invalidisierenden subtalaren Arthrosen führen [16]. Je nach Ausmaß der Deformität resultieren weitere Probleme wie eine Irritation der Peronealsehnen oder das Anstoßen der Fersenbeinwand an die Spitze der Außenknöchels (Abutment) [11,24]. Daraus ergibt sich die unbedingte Forderung nach exakter Verletzungsanalyse und Indikationsstellung zur anatomischen Reposition, um schwere Folgeschäden zu vermeiden.

\section{Anatomie}

Das Fersenbein (griech. Calx, lat. Calcaneus, Os calcis) ist der größte Fußwurzelknochen und bildet den Abschluss der lateralen Fußsäule. Als hinterer Abschnitt des Fußlängsgewölbes wirkt er als kräftiger Hebelarm an dem mit der Achillessehne die kräftigste Sehne des Körpers ansetzt. Daraus ergibt sich eine wesentliche Bedeutung für die Statik und Dynamik des gesamten Fußes, welche bei dislozierten Fersenbeinbrüchen empfindlich gestört wird. Das Fersenbein leitet die axial einwirkenden Kräfte vom Sprungbein auf das vordere und hintere 


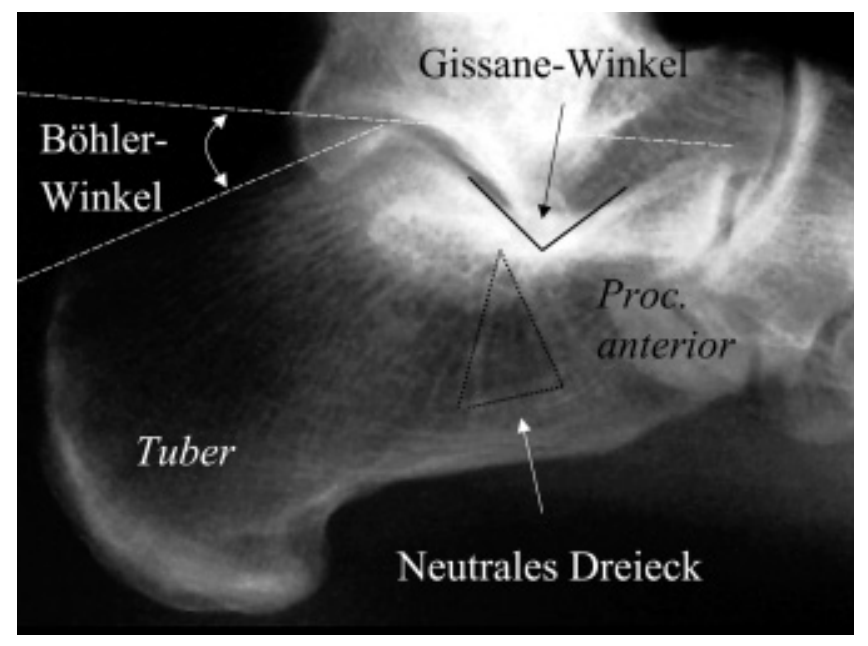

Fußlängsgewölbe weiter, woraus sich die typische Spongiosastruktur mit fächerförmig auslaufenden Lastträgern, den Trabekeln, ableitet. Dadurch entsteht unterhalb des Fersenbeinhalses eine wenig belastete Zone, das sogenannte neutrale Dreieck, mit nur spärlicher Trabekelstruktur (Abb.1). Die Schale aus kortikalem Knochen ist ebenfalls von unregelmäßiger Dicke, eine Schwachstelle stellt hier die seitliche Wand des Fersenbeines dar. Die kräftigste Spongiosa findet sich in Gebieten höherer Lastaufnahme, also unterhalb der Gelenkfläche zum Talus, die als „Thalamus calcanei“ bezeichnet wird, im Fersenbeinhöcker (Tuber calcanei), am Sustentaculum tali sowie im Bereich des Gissane'schen Winkels (Abb.1). Das Sustentaculum tali bildet einen biomechanisch stabilen Fortsatz, der das Sprungbein medialseitig abstützt und über kräftige Bänder mit diesem verbunden ist. Der ebenfalls kräftige Processus anterior calcanei stellt mit seiner Gelenkfläche zum Würfelbein (CalcaneocuboidGelenk) die Verbindung zum ChopartGelenk, und somit zum Mittelfuß her. Die Trochlea peronealis auf der ansonsten glatten und dünnwandigen Außenseite des Calcaneus bildet das biomechanisch wichtige Gleitlager für die Peronealsehnen. Drei der vier Gelenkflächen des Fersenbeines führen zum Talus, eine zum Cuboid. Die größte und funktionell entscheidende posteriore Gelenkfacette bildet mit der korrespondierenden Gelenkfläche des Talus das hintere untere Sprunggelenk. Die mediale und anteriore Gelenkfacette, welche in $20 \%$ der Fälle verschmolzen sind, liegen im vorderen Anteil des unteren Sprunggelenkes (Art. talocalcaneonaviculare).
Abb. 1 Anatomie des Fersenbeines. Der Verlauf der Spongiosabälkchen (Trabekulation) gibt die Richtung der Krafteinleitung vom Talus auf den Calcaneus wieder.

Die komplizierte anatomische Form des Fersenbeines leitet sich aus seiner biomechanischen Funktion als Abschluss des Fußlängsgewölbes und der lateralen Fußsäule $a b$ und spiegelt den Weg der Kraftfortleitung vom Sprungbein auf den Fuß wider.

Ein wesentliches Kriterium für die anatomische Form und damit Richtlinie für die operative Aufrichtung von Fersenbeinfrakturen ist der sogenannte Tuber-Gelenk-Winkel („Böhler-Winkel“), welcher von zwei gedachten Linien von der Oberkante des Tuber calcanei und des Calcaneocuboid-Gelenkes in Projektion auf den Hinterrand des Subtalargelenkes gebildet wird [3]. Dabei wird als Winkelmaß der Komplementärwinkel zu $180^{\circ}$ angegeben, welcher im Normalfall zwischen 25 und $40^{\circ}$ liegt (Abb.1).

\section{Frakturmechanismus}

Fersenbeinfrakturen entstehen typischerweise durch axiale Gewalteinwirkung beim Sturz aus der Höhe oder beim Verkehrsunfall (Dezeleration), wobei sich der kräftige Processus lateralis tali in die posteriore Gelenkfacette des Calcaneus einstaucht. Aufgrund der seitlich zueinander versetzten Vertikalachsen von Talus und Calcaneus entsteht zunächst eine Scherfraktur zwischen dem tuberculumtragenden Hauptfragment des Calcaneus und dem Sustentaculum tali (Abb.2). Klassischerweise kommt es zur Ausbildung einer primären Frakturlinie (Abb.3), welche am Vorderrand der posterioren Gelenkfacette beginnt [7]. Befindet sich der Fuß beim Aufprall in einer Supinationsstellung, liegt diese Frakturlinie weit medial, so dass gelegentlich isolierte extraartikuläre Fraktu-

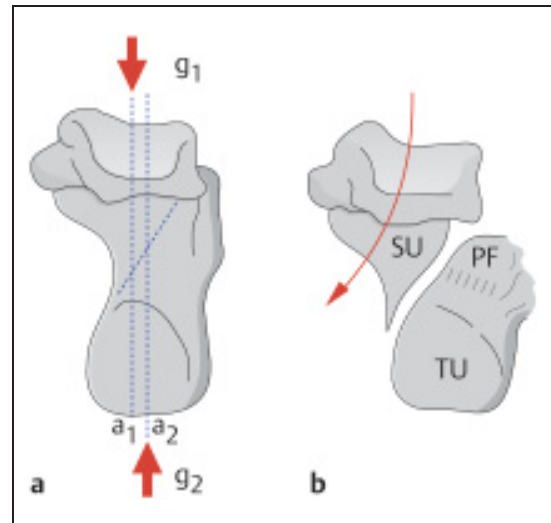

Abb. 2 Mechanismus der Entstehung von Calcaneusfrakturen durch axiale Gewalteinwirkung. Die typische Abscherung des sustentakulumtragenden Hauptfragmentes (SU) gegen die Tuberositas (TU) entsteht durch die gegeneinander versetzten Vertikalachsen von Talus (a1) und Calcaneus (a2).

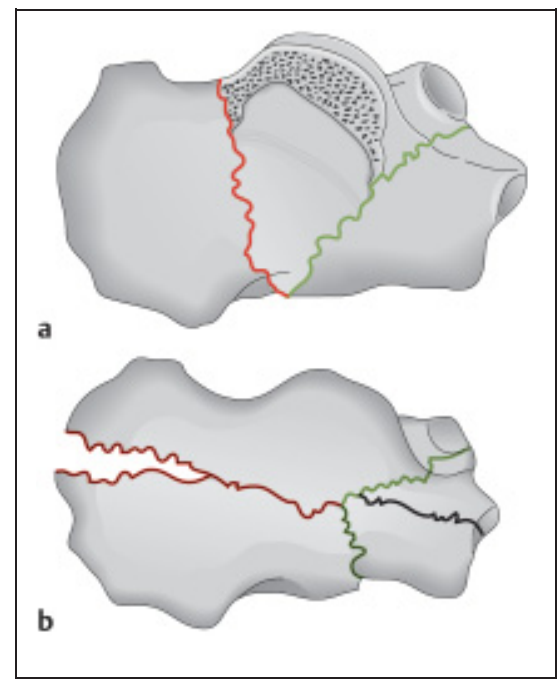

Abb. 3 Die primäre Frakturlinie (grün) beginnt regelhaft am Vorderrand der posterioren Gelenkfacette. Je nach Verlauf der sekundären Frakturlinie (rot) entsteht das typische Erscheinungsbild einer joint depression- (a) oder tongue type Fraktur (b) nach Essex-Lopresti [7].

ren des Sustentaculum tali entstehen [24]. In Abhängigkeit von der Energie des Aufpralles und der Qualität des Knochens entstehen sekundäre und tertiäre Frakturlinien, so dass bis zu fünf Hauptfragmente entstehen können. Nach CTAnalyse von über 200 Frakturen wurden in $36 \%$ vier und in $58 \%$ fünf Hauptfragmente gesehen. Über drei Viertel aller Calcaneusfrakturen sind intraartikulär, davon weisen $97 \%$ Verwerfungen der posterioren Facette, 59\% zusätzlich des Calcaneocuboidgelenkes und $8 \%$ der anterioren Facette auf [24]. 
Durch die spongiöse Binnenstruktur und die Beteiligung an vier Gelenkflächen entstehen am Calcaneus im Vergleich zu anderen Fußknochen häufiger Frakturen mit Gelenkbeteiligung, eingestauchte Frakturen und Trümmerbrüche.

Beim Jugendlichen mit noch elastisch verformbarem Calcaneus entstehen beim Sturz aus der Höhe durch die reflektorische Aktion des M. triceps surae eher die typischen extraartikulären Abrissfrakturen des Achillessehnenansatzes (sog. „Entenschnabelbrüche“). Bei heftigen Distorsionen kann es entlang der Hellpapp'schen Supinationslinie zu einem knöchernen Abriss des Lig. bifurcatum aus dem Proc. anterior calcanei kommen.

\section{Frakturklassifikation}

Aus den charakteristischen sekundären Frakturlinien im Tuber calcanei leitet sich die klassische Fraktureinteilung von Essex- Lopresti in „,joint-depression-“ und „tongue-type“ ab [7]. Bei der ,joint-depression“-Fraktur ist hauptsächlich die posteriore Facette imprimiert und die Frakturlinie verläuft unmittelbar dahinter in den Fersenbeinhöcker hinein. Tritt diese nach langstreckigem Verlauf vor dem Achillessehnenansatz aus, spricht man aufgrund der Form des entstehenden langgezogenen Fragmentes, welches durch den Zug der Achillessehne nach proximal gezogen wird, vom „tongue type“ (Abb.3).

Tertiäre Frakturlinien können durch den Processus anterior verlaufen, so dass bis zu 5 Hauptfragmente resultieren [23]. Diese bilden die Grundlage einer auf CT-Schnitten basierenden Klassifikation, welche ebenso die Beteiligung von maximal 3 Gelenkfacetten (anteriore und mediale Facette werden hierfür zusammengefasst) berücksichtigt (Abb.4). Die additive Graduierung des Weichteilschadens bei I.-III. ${ }^{\circ}$ offenen und geschlossenen Frakturen (1 - 3 Punkte) sowie die Frakturen benachbarter Knochen oder Trümmerfrakturen eines der Hauptfragmente (Zusatzpunkt) kann zu einer Summation von maximal 12 Punkten führen (Tab.1). Diese Frakturskala hat einen prognostisch prädiktiven Wert von 86\% [24].

Die im amerikanischen Raum bevorzugt verwendete Klassifikation von Sanders [18] basiert auf der für das therapeutische Vorgehen und funktionelle Ergebnis entscheidenden Anzahl der Frakturlinien im koronaren CT-Schnitt auf Höhe der poste-

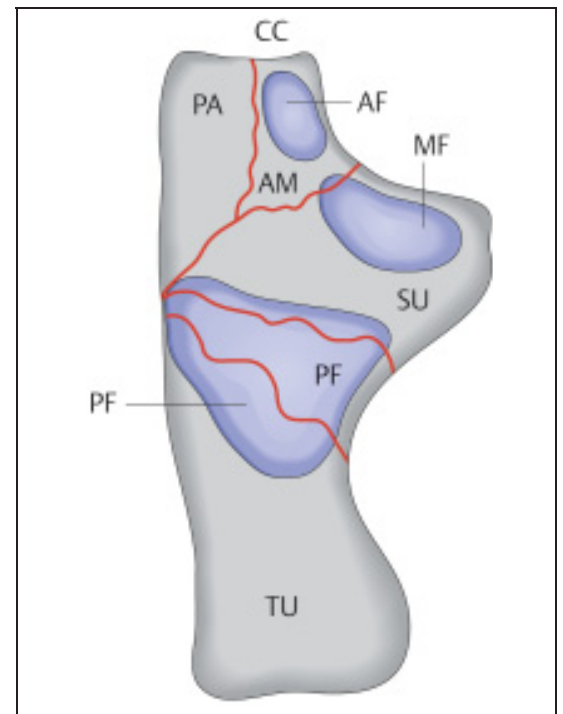

Abb. 4 Die 5 Hauptfragmente (TU = Tuberositas, SU = Sustentaculum tali, PF = posteriore Facette, $\mathrm{PA}=$ Proc . anterior und $\mathrm{AM}=$ anteromediales Facettenfragment) und 3 Gelenkfacetten (posteriore Facette $=\mathrm{PF}$, anteriore $/ \mathrm{me}$ diale Facette $=A F+M F$, Calcaneocuboid-Gelenk $=\mathrm{CC}$ ) dienen als Basis für die Frakturklassifikation [24].

rioren Facette. Alle nichtdislozierten Frakturen werden unabhängig von der Anzahl der Frakturlinien Typ I zugeordnet. Typ II weist eine, Typ III zwei, Typ IV (Trümmerfraktur) 3 und mehr Frakturlinien in der posterioren Facette auf.

\section{Diagnostik}

\section{Klinische Diagnostik}

Die klinische Diagnose weist nicht nur den Weg zu den erforderlichen bildgebenden Verfahren, sondern erfasst auch die für das weitere Vorgehen und die Prognose wichtige Graduierung des primären Weichteilschadens.

Tab. 1 Calcaneus-Frakturskala (Zwipp 1994)

x Fragmente

y Gelenke

Weichteilschaden $\left(I-I I I^{\circ}\right.$ geschlossen oder offen)

Trümmerfraktur eines Hauptfragmentes, regionale Zusatzfraktur

gesamt
Typischerweise findet sich eine druckschmerzhafte Schwellung mit begleitendem Hämatom über der gesamten Fersenregion. Der betroffene Fuß kann nicht belastet und nicht schmerzfrei pro- oder supiniert werden. Bei erheblicher Schwellung muss immer ein akutes Kompartmentsyndrom, gegebenenfalls mit Druckmessung ausgeschlossen werden. Nicht selten finden sich Spannungsblasen über dem Rückfuß. Frakturen ohne ersichtliche Dislokation oder Weichteilschäden werden nach wie vor gelegentlich als Sprunggelenksdistorsion fehldiagnostiziert und insbesondere bei polytraumatisierten oder mehrfachverletzten Patienten initial leicht übersehen, wenn andere Verletzungen im Vordergrund stehen.

\section{Bildgebende Diagnostik}

Die klinische Verdachtsdiagnose wird radiologisch durch konventionelle Röntgenaufnahmen in 3 Standardebenen (Calcaneus lateral und axial, Fuß dorsoplantar) gesichert. Ergänzend wird immer eine OSG Map-Aufnahme sowie zur Darstellung des Subtalargelenkes eine Brodén-Serie durchgeführt. Bei klarer OP-Indikation sollte präoperativ zum Vergleich eine seitliche Aufnahme des kontralateralen Fußes angefertigt werden, da der Böhler-Winkel, und somit das anzustrebende Repositionsergebnis, einer erheblichen interindividuellen Schwankung unterliegt. Unabdingbare Voraussetzung für die Frakturklassifikation und präoperative Planung ist die Durchführung eines CT mit axialer und koronarer Schnittführung $[18,24]$, während das MRT keine therapierelevanten Zusatzinformationen erbringt und daher nicht routinemäßig empfohlen wird. 


\section{Therapie}

\section{OP-Indikation und -Zeitpunkt}

Die Indikation zum operativen Vorgehen besteht bei allen intraartikulären Fersenbeinfrakturen mit $>1 \mathrm{~mm}$ Gelenkstufe sowie bei extraartikulären Frakturen mit relevanter Rückfußfehlstellung (Varus $>5^{\circ}$, Valgus $>10^{\circ}$ ),

sofern keine Kontraindikationen vorliegen [24]. Lokale Kontraindikationen zur Osteosynthese bestehen bei kritischen Weichteilverhältnissen mit hohem Infektionsrisiko wie Spannungsblasen, Hautnekrosen sowie bei fortgeschrittenen arteriellen bzw. venösen Durchblutungsstörungen. Systemische Kontraindikationen sind der juvenile insulinpflichtige Diabetes mellitus, die manifeste HIV-Infektion bzw. Immunosuppression sowie eine fehlende Compliance von Seiten des Patienten (wie z.B. bei Alkoholund Drogenabhängigen). Ein höheres biologisches Alter von ( $>65$ Jahren) stellt lediglich eine relative Kontraindikation dar, hier steht der individuelle Aktivitätsgrad und Funktionsanspruch im Vordergrund.

Aufgrund der zumeist erheblichen Weichteilschwellung liegt der Operationszeitpunkt bei $\mathrm{I}^{\circ}$ und $\mathrm{II}^{\circ}$ ig geschlossenen Frakturen im Allgemeinen zwischen dem 6.und 10.Tag nach dem Trauma. In der Zwischenzeit wird der betroffene Fuß durch lokale und systemische Maßnahmen (Hochlagerung, Eisapplikation bzw. cryo-cuff ${ }^{\circledR}$, aktive Venenpumpe, Lymphdrainage, Antiphlogistika, enzymatische Präparate) zum Abschwellen gebracht. Eine verzögerte Versorgung nach mehr als 14 Tagen nach dem Unfallereignis, insbesondere bei stärkerer Dislokation, ist mit einer potentiell erhöhten postoperativen Infektionsgefahr behaftet und sollte daher vermieden werden [25].

Die notfallmäßige OP-Indikation besteht bei offenen Frakturen, inkarzerierten Weichteilen sowie manifestem Kompartment-Syndrom.

Offene Fersenbeinfrakturen stellen absolute Problemverletzungen dar, deren Behandlung spezialisierten Zentren vorbehalten bleiben sollte. Die Notfallversorgung besteht in einem initialen Wunddébridement, der Reposition und temporären minimal-invasiver Osteosynthese mit perkutan eingebrachten Kirschner-Drähten oder einer medialen Transfixation (mit je einer Schanz-Schraube in distaler

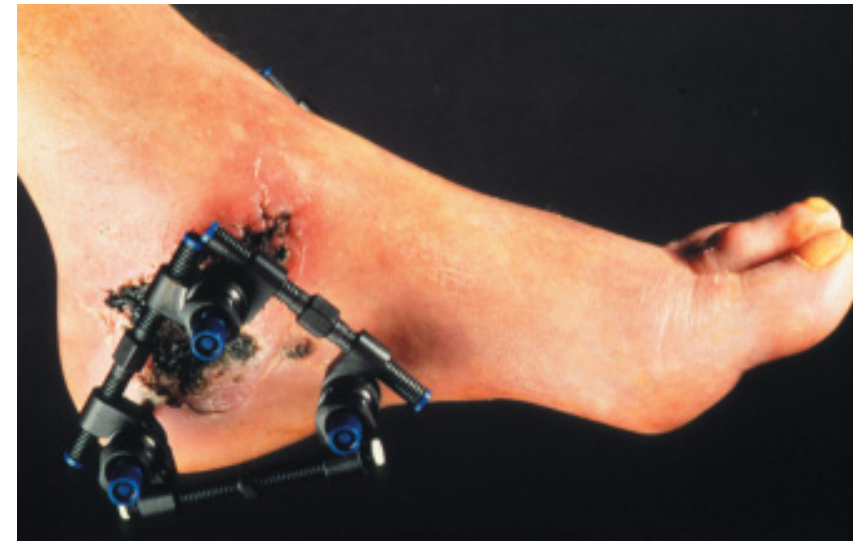

Abb. 5 Mediale Dreipunkt-Fixation bei offenen Frakturen oder geschlossenen Frakturen mit erheblichem Fragmentdruck auf die Weichteile: Prototyp eines röntgenstrahlendurchlässigen Distraktors.

Tibia, Tuber calcanei und Metatarsale I bzw. als medialer Dreipunkt-Fixateur im Calcaneus, s. Abb.5) sowie der temporären Kunsthautdeckung. Eine Secondlook-Operation muss regelhaft innerhalb von 48-72 Stunden erfolgen, zu diesem Zeitpunkt sollte idealerweise bereits die definitive Osteosynthese und Weichteildeckung durchgeführt werden. Bei definitiver Osteosynthese im Intervall von etwa einer Woche ist selbst bei korrektem Vorgehen die Infektionsrate von II.-III. ig offenen Frakturen etwa um das 5-fache gegenüber geschlossenen Frakturen erhöht [17]. Die Indikation zur frühen Lappendeckung sollte daher großzügig gestellt werden, und hat bei komplexen Fußverletzungen mit höhergradigem Weichteilschaden zu einem Rückgang der schicksalhaften Infekte geführt [4]. Nicht praktikabel ist dies bei polytraumatisierten Patienten, bei denen lebenserhaltende Maßnahmen im Vordergrund stehen und Patienten, deren eingeschränkter Allgemeinzustand längere Eingriffe nicht zulässt.

Bei geschlossenen Frakturen mit erheblichem Fragmentdruck auf die Weichteile kann notfallmäßig eine geschlossene Reposition versucht werden, was jedoch nur selten gelingt. Meist ist eine perkutane Reposition mit einer in das Tuber calcanei eingebrachten Schanz-Schraube erforderlich. Die Hauptfragmente werden gegebenenfalls minimal-invasiv mit Kirschner-Drähten fixiert, das Hämatom über Stichinzisionen abgesaugt. Das Repositionsergebnis wird durch einen medialen Dreipunkt-Fixateur (Abb.5) gesichert, um nach Ablauf von 8-10 Tagen die definitive Versorgung, wie unten geschildert, durchzuführen [24]. Bei III ${ }^{\circ}$ ig geschlossenen Frakturen mit manifestem Kompartment-Syndrom erfolgt beim Polytrauma die notfallmäßige Dermatofasziotomie über einen ausgedehnten dor- so-medianen Zugang, mit Anlage eines medialen Fixateurs, beim Monotrauma die sofortige operative Versorgung mit definitiver Plattenosteosynthese.

\section{Konservative Therapie}

Die konservativ-funktionelle Therapie ist bei extraartikulären Frakturen ohne relevante Fehlstellung, den seltenen undislozierten intraartikulären Frakturen sowie bei bestehenden Kontraindikationen zur operativen Therapie angezeigt. Das Ziel ist die frühe Mobilisation des Patienten nach initialer Abschwellung der Weichteile, was durch kurzzeitige Bettruhe (3-4 Tage) mit begleitenden antiphlogistischen Medikamenten, lokaler Kühlung und späterhin Lymphdrainage erreicht wird. Nach dieser Zeit beginnt die aktive Beübung der Sprunggelenke und die Mobilisation mit Abrollen des betroffenen Fußes unter Entlastung für 3-6 Wochen. Die Vollbelastung wird abhängig von der Knochenqualität und Frakturanatomie nach 6-12 Wochen erreicht. In speziell angefertigtem Schuhwerk mit Abstützung am Tibiakopf (z.B. nach Pässler) ist die Vollbelastung unter Umständen bereits nach 8-10 Tagen erlaubt.

Besteht bei jungen Patienten mit absoluter Kontraindikation zur operativen oder semioperativen Behandlung eine ausgeprägte Fehlstellung des Rückfußes, so kann das geschlossene Repositionsmanöver nach Omoto et al. [14] durchgeführt werden, welches allerdings von intakten talocalcanearen Bändern zum Erhalt der Retention abhängig ist. Der Patient befindet sich in Bauchlage mit rechtwinklig gebeugtem Knie, in Regional- oder Allgemeinanästhesie. Die Reposition beginnt mit einem Lösen der Fragmente unter ruckartiger Varisierung oder Valgisierung des Rückfußes bei ständiger bilateraler Kompression der Ferse unter maximalem 
axialen Zug. Das Repositionsergebnis wird mittels Bildwandler kontrolliert. Unter permanentem Zug und bimanueller Kompression wird ein zirkulärer Unterschenkelgips angelegt, welcher je nach Frakturschwere für 3-6 Wochen belassen wird. Die Entlastungsdauer entspricht den oben genannten Perioden. Die günstigsten Ergebnisse werden bei möglichst früher Reposition erreicht, was jedoch aufgrund der Weichteilschwellung in den wenigsten Fällen möglich ist. Zudem geht nach Abschwellung der Weichteile das ohnehin oft unvollständige Repositionsergebnis zumeist verloren.

\section{Semi-operative Therapie}

Die heute praktizierten semi-operativen Methoden gehen im Wesentlichen auf die von Westhues 1934 entwickelte Aufrichtung des Tuber calcanei mit einer perkutan eingebrachten Schanz-Schraube zurück. Die Fixation, von Westhues [22] und später Essex-Lopresti [7] im Gips durchgeführt, erfolgt heute mit perkutan eingebrachten Kirschner-Drähten oder Schrauben bzw. einem Fixateur-System, wie z.B. dem medialen 3-Punkt-Distraktor (Abb.5). Diese Verfahren dienen mehrheitlich der Stabilisierung dislozierter Frakturen beim jungen Patienten mit lokaler oder allgemeiner Kontraindikation zum offenen Vorgehen oder geschlossenen Frakturen beim Polytrauma mit innerem Druck der Fragmente auf die Weichteile. Bei offenen Frakturen erlauben sie eine temporäre Fixation der Fragmente bis zur definitiven Versorgung. In neuerer Zeit werden zur Weichteilschonung perkutane Osteosyntheseverfahren unter Bildwandlerkontrolle auch bei speziellen Frakturtypen mit einer Frakturlinie im Subtalargelenk (Sanders II) favorisiert [21]. Im eigenen Vorgehen wurde bei bislang 15 Patienten mit Sanders-II-Frakturen eine perkutane, arthroskopisch assistierte Schraubenosteosynthese durchgeführt, die ersten 1 -jahres-Ergebnisse sind exzellent [9].

\section{Operative Therapie}

Ziel der operativen Versorgung von Fersenbeinfrakturen ist die anatomische Rekonstruktion von äußerer Form und Gelenkkongruenz mittels primärer, übungsstabiler und nicht gelenkübergreifender Osteosynthese.

Calcaneusfrakturen werden üblicherweise in Intubationsnarkose und optional in regionaler Blutsperre versorgt. Bei den relativ seltenen extraartikulären 2- und 3-Fragment-Brüchen wird ein medialer Zugang (nach McReynolds, 6) gewählt. Der Patient befindet sich in Rückenlage. Die Inzision erfolgt auf einer Länge von $8-10 \mathrm{~cm}$ parallel zur Fußsohle exakt auf halber Strecke zwischen Malleolus medialis und medialem Rand der Fußsohle. Unter stumpfem Weghalten des tibialen Gefäß-Nerven-Bündels wird das sustentakulumtragende Fragment dargestellt. Die Flexor-hallucis-longus-Sehne wird identifiziert und in situ belassen. Das tuberositäre Fragment wird über eine perkutan eingebrachte Schanz-Schraube zum sustentakulären Fragment reponiert. Ein eventuell vorhandenes Processus-anterior-Fragment wird zur medialen Wand des sustentakulären Fragmentes bündig reponiert und ebenfalls mit temporären Kirschner-Drähten gehalten. Anschließend wird das sustentakulumtragende Fragment mittels eines kleinen H-Plättchens, im Sinne eines medialen Antigleit-Plättchens, gegen das tuberositäre und Processus-anterior-Fragment retiniert. Extraartikuläre Frakturen des Tuber calcanei wie z.B. die sogenannten „Entenschnabelbrüche“ können mit Schrauben über Stichinzision versorgt werden.

Intraartikuläre Fersenbeinfrakturen werden bevorzugt über den ausgedehnt lateralen Zugang versorgt. Dieser berücksichtigt die Blutversorgung des Rückfußes und gewährt eine ausreichende Übersicht vom subtalaren Gelenkspalt bis zum Calcaneocuboid-Gelenk.

Der Eingriff erfolgt in Seitenlage. Der Hautschnitt liegt bumerangförmig zwischen Außenknöchel und Achillessehne bzw. lateralem Fußsohlenrand (Abb.6). Neuere anatomische Studien zur Blutversorgung des lateralen calcanearen Hautlappens haben gezeigt, dass die ideale Schnittlinie nicht exakt auf halber Strecke, sondern mehr in Richtung auf die Achillessehne bzw. Fußsohle verlaufen sollte (im eigenen Vorgehen auf ca. $2 / 3$ der Strecke). Dies entspricht dem Versorgungsgebiet der A. calcanea lateralis und dient der Schonung des N. suralis sowie der V. saphena parva [8]. Anschließend erfolgt die subperiostale einschichtige Anhebung eines lateralen Haut-Weichteil-Lappens unter Schonung der Hautnervenäste ( $\mathrm{N}$. cutaneus dorsalis intermedius et lateralis) mit scharfer periostaler Ablösung des Lig. fibulocalcaneare und des distalen peronealen Retinaculums. Bei Beteiligung des Calcaneocuboidgelenkes werden die Peronealseh-

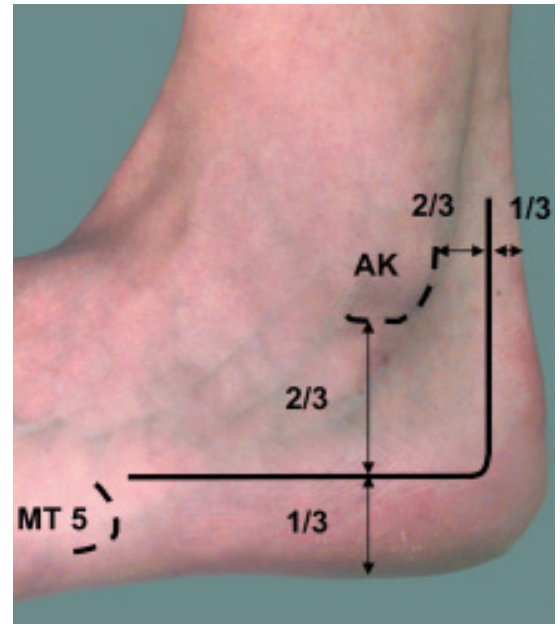

Abb. 6 Erweiterter lateraler Zugang modifiziert nach Benirschke et al. (extended lateral approach, 1).

nen weit nach distal mobilisiert, wobei die Sehnenscheide geschlossen bleibt.

Die Aufrichtung und Achsenkorrektur des tuberositären Hauptfragmentes erfolgt wiederum durch das Repositionsmanöver nach Westhues mit Einbringen einer Schanz-Schraube in das Tuber calcanei (Abb. 7a). Durch axialen Zug und Valgisation kann die regelhaft impaktierte posteriore Facette dargestellt werden, wobei zur besseren Übersicht das laterale Wandfragment türflügelartig weggeklappt werden muss. Ist das sustentakulumtragende Fragment in Relation zum Talus gekippt, muss dieses zunächst reponiert und temporär kongruent gegen den Talus fixiert werden. Der Aufbau der posterioren Gelenkfacette erfolgt nun schrittweise von medial nach lateral. Die Fragmente werden mit 2 parallel zur Gelenkfläche eingebrachten Kirschnerdrähten gegeneinander fixiert. Anschließend wird das tuberositäre Fragment mittels der eingebrachten SchanzSchraube gegen das Proc.-anteriorFragment reponiert, wodurch der Böhler-Winkel wiederhergestellt wird (Abb. 7b). Die erreichte Position wird nun durch 2-3 Kirschner-Drähte, welche von posterolateral, d.h. vom tuberositären Fragment durch das posteriore Facettenfragment in den Talus eingebracht werden sowie durch 2 weitere Drähte, welche vom Cuboid aus durch das Proc.-anterior-Fragment in den Tuber calcanei eingebracht werden, fixiert. Zuvor ist jedoch die Kongruenz im Calcaneocuboid-Gelenk zu überprüfen (Abb.8).

Verbleiben nach der Reposition in der Stauchungszone Knochendefekte von 


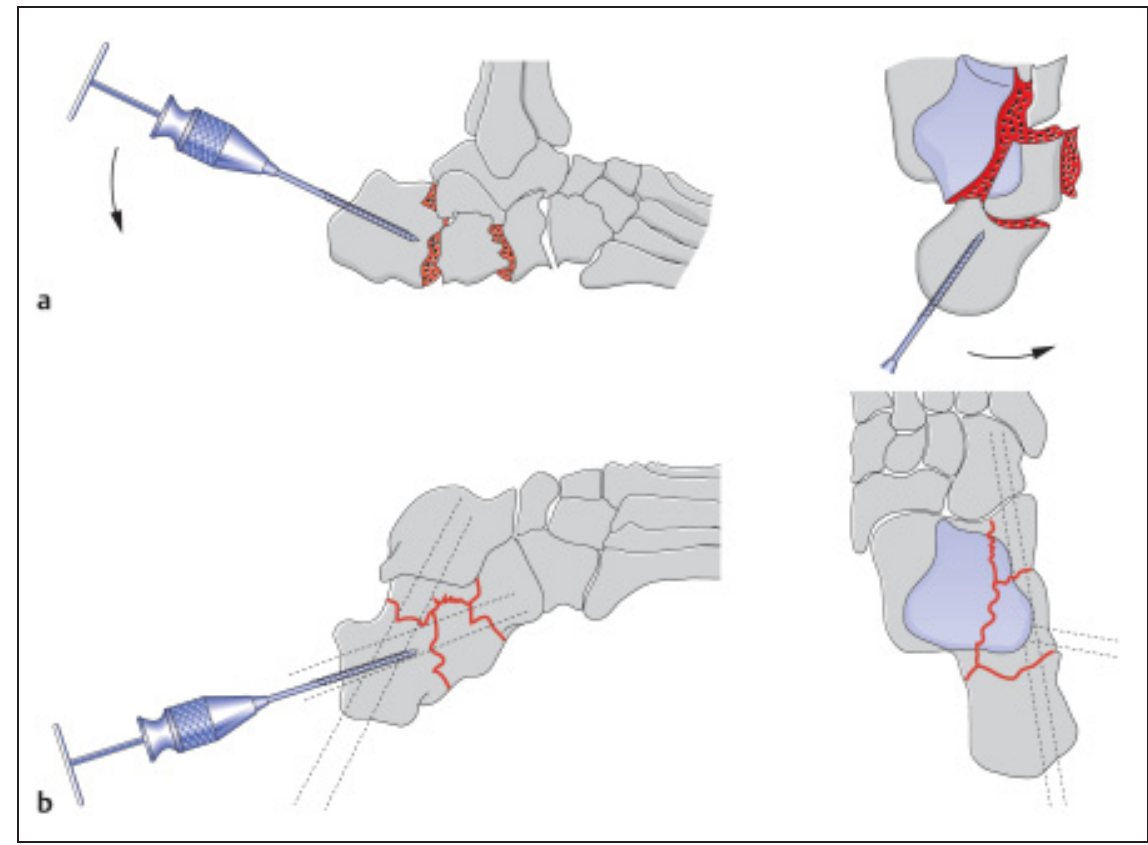

Abb. 7 Mit einer perkutan in das tuberositäre Fragment eingebrachten Schanz-Schraube wird sowohl das Fersenbein aufgerichtet als auch begleitende Varus- bzw. Valgus-Fehlstellungen ausgeglichen (Westhues-Manöver, 22).

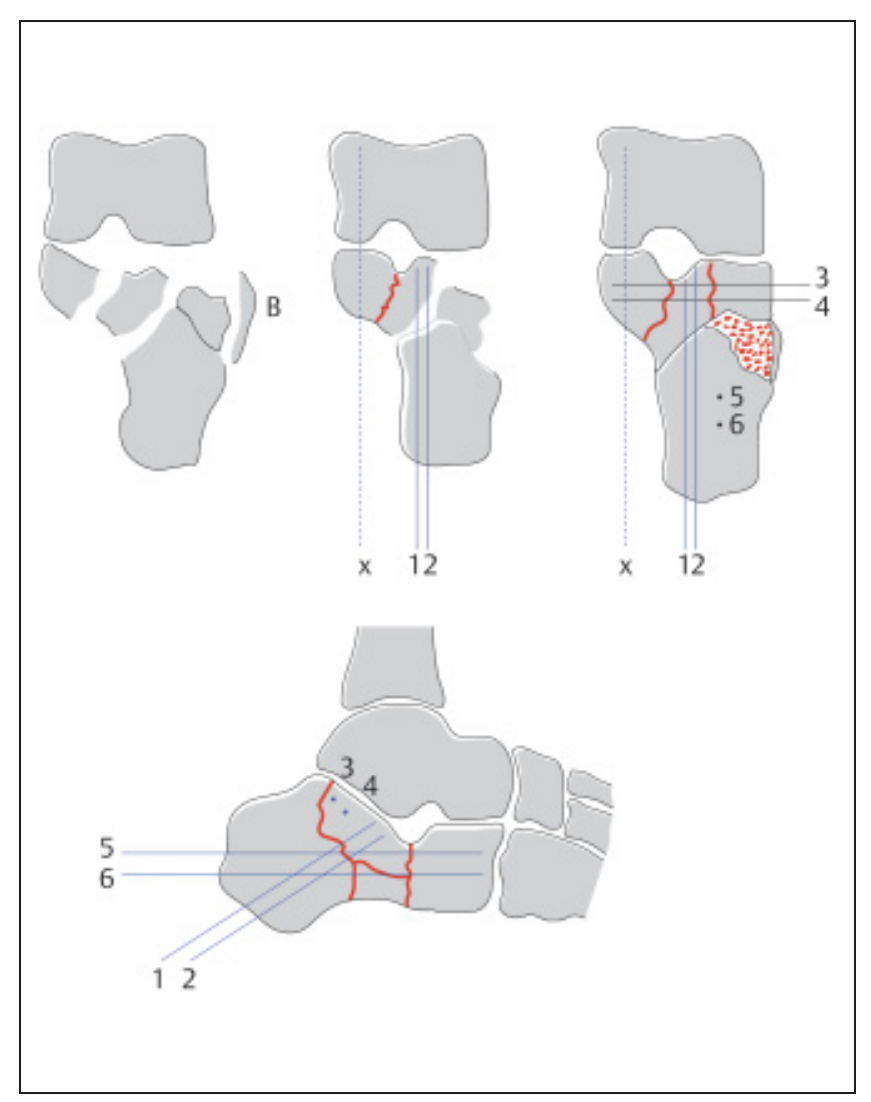

mehr als Daumenendgliedgröße wird von einigen Autoren eine Unterfütterung mit autologer Spongiosa vom ipsilateralen Beckenkamm durchgeführt, bevor das zuvor weggeklappte Wandfragment zurückgeschlagen wird, welches sich nun exakt einpassen lassen muss $[1,13,23]$. Die Notwendigkeit der Unterfütterung solcher Stauchungsdefekte wird jedoch kontrovers diskutiert und von anderen Autoren nicht gesehen $[12,18]$. Der Einsatz von Knochenersatzstoffen anstelle autologer Spongiosa ist noch nicht als etabliert anzusehen.

Nach erfolgter Reposition wird im eigenen Vorgehen die Kongruenz der visuell schlecht einsehbaren posterioren Facette mittels offener Arthroskopie des subtalaren Gelenkes kontrolliert [16]. Dazu wird ein Arthroskop mit kleinem Durchmesser, ggf. Winkeloptik, in den freiliegenden subtalaren Gelenkspalt eingeführt. Unter arthroskopischer Sicht können jetzt Feinkorrekturen und Nachrepositionen bei verbliebenen Inkongruenzen in der posterioren Gelenkfacette erfolgen, welches nach ersten Erfahrungen in etwa einem Viertel der Fälle notwendig wird [Abb.9]. Dieses Verfahren erfordert keinen großen Zeitaufwand und ist bezüglich Präzision und Gründlichkeit der Bildwandlerkontrolle bzw. der direkten Sicht deutlich überlegen. Die Retention erfolgt mehrheitlich mit einer der Anatomie des Calcaneus angepassten AO-Platte nach Sanders [19], es sind jedoch verschiedene, meist Y-förmige Platten, im Gebrauch. Seit dem vergangenen Jahr steht auch ein winkelstabiles Implantat der $\mathrm{AO} /$ ASIF Foot and Ankle Expert Group zur Verfügung, welches sich an einigen Zentren in der klinischen Erprobung befindet. Die Plattenfixation an der lateralen Fersenbeinwand (Abb. 10) wird in der Regel mit sechs 3,5-mm-Kortikalisschrauben erreicht, davon 2 unterhalb der subtalaren Gelenkfläche in Richtung auf das Sustentaculum tali, 2 gelenknah im Processus anterior und 2 in das Tuber cacanei $[1,24]$. Abschließend wird die Röntgenkontrolle in 3 Standardebenen (Fuß seitlich und dorsoplantar, Calcaneus axial) sowie eine $20^{\circ}$ Brodèn-Spezialaufnahme durchgeführt. Der Hautverschluss erfolgt schichtweise unter Einlegen einer Redondrainage.

Ein bilaterales Vorgehen ist in den seltensten Fällen erforderlich und mit einer erhöhten Rate an Wundrandnekrosen verbunden. Besteht jedoch eine Fraktur des Sustentaculum tali bis in die mediale Gelenkfacette hinein, welche sich 

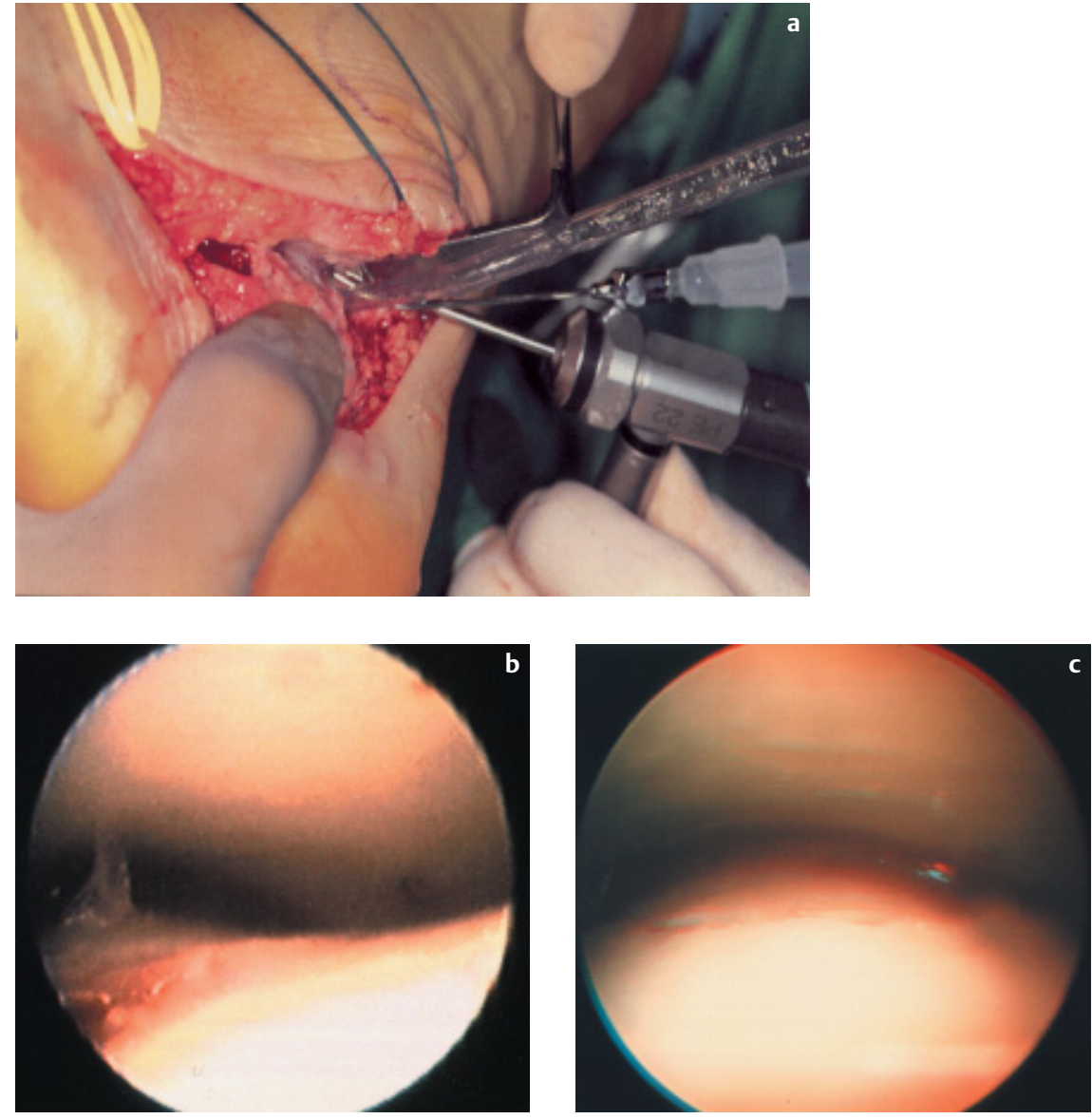

Abb.9 Offene subtalare Arthroskopie zur Kontrolle des Repositionsergebnisses mit dem MiniArthroskop unter manueller Spülung (a). Die nach erfolgter Reposition unter Bildwandlerkontrolle arthroskopisch noch sichtbare Stufe beträgt aufgrund des Vergrößerungsfaktors nur ca. $1 \mathrm{~mm}$, kann jedoch zu erheblichen arthrotischen Beschwerden führen (b). Daher erfolgt eine Feinkorrektur, die anschließende Kontrollarthroskopie zeigt einen stufenlosen Verlauf auch des schlecht einsehbaren medialen Anteiles der posterioren Gelenkfacette.

von lateral nicht anatomisch reponieren lässt, so ist zusätzlich zum ausgedehnt lateralen Zugang eine kleine, querverlaufende Inzision direkt über dem tastbaren Sustentaculum tali erforderlich (Sustentaculum-Zugang nach Zwipp, 24). In einem ersten Operationsschritt wird unter Schonung der Sehnen des M. tibialis posterior, M. flexor digitorum longus und $\mathrm{M}$. flexor hallucis longus zunächst das Sustentaculum gegen die mediale $\mathrm{Ge}$ lenkfacette des Talus reponiert mit zwei Kortikalisschrauben retiniert, um anschließend mit der Versorgung von lateral fortzufahren. In diesen Fällen ist die Lagerung des Patienten in Rückenlage mit Keilanhebung der betroffenen Seite und beiderseitigen Stützen empfehlenswert.

Verschiedene Autoren sehen bei Trümmerfrakturen mit irreparabler Schädigung der posterioren Gelenkfacette die Indikation zur primären subtalaren $\mathrm{Ar}$ - throdese. Während Sanders [19] zunächst eine Standard-Osteosynthese des Calcaneus durchführt um anschließend das Subtalargelenk mit einer Spongiosazugschraube zu fusionieren, praktizieren Buch et al. [5] eine reine primäre Schraubenarthrodese. Im eigenen Vorgehen wird generell die primäre Rekonstruktion angestrebt, um eine exzessive Weichteilkontraktur zu vermeiden und eine eventuelle später notwendige Arthrodese zu erleichtern. Diese erfolgt sekundär bei entsprechendem Beschwerdebild als Insitu-Arthrodese, in einigen Fällen wurde auch die Ausbildung einer symptomarmen Ankylose gesehen, welche kein weiteres operatives Vorgehen erforderte. Die Rate sekundärer subtalarer Arthrodesen liegt bei diesem Vorgehen zwischen 2,3 und $3.7 \%[1,24,25]$.

Die Nachbehandlung erfolgt frühfunktionell aus dem Unterschenkelspaltgipsverband heraus mit krankengymnastischen
Übungen ab dem 1.postoperativen Tag. Diese beinhalten geführte, aktive Flexions/Extensionsbewegungen. Zum Leerpumpen der venösen Plexus wird der Patient zum regelmäßigen Fußdruck gegen die Gipssohle angehalten. Am 2. postoperativen Tag beginnt der Patient mit aktiven Kreiselbewegungen des Fußes sowie passiven Bewegungen auf der Motorschiene. Die Mobilisation erfolgt ab dem 5.-8. postoperativen Tag unter Teilbelastung von $15 \mathrm{kp}$. Auf Vollbelastung kann abhängig von Bruchform, Knochenqualität und Ausmaß einer eventuell notwendigen Spongiosaimplantation nach 6-12 Wochen übergegangen werden. Schwerstarbeit und Sport sind in der Regel nach 4-6 Monaten möglich. Die Implantatentfernung wird nach einem Jahr empfohlen (Abb.11), ist jedoch nicht obligat. Bei deutlicher Rückfußeinsteifung sollte diese aufgrund der nach eigener Erfahrung regelhaft beobachteten fibrösen Verwachsungen immer mit einer lateralseitigen Arthrolyse und Arthroskopie des subtalaren Gelenkes verbunden werden [16,25].

\section{Komplikationen}

Aseptische Wundrandnekrosen treten in 2 bis $10 \%$ der Fälle auf und können durch lokal-desinfizierende Maßnahmen zum Ausheilen gebracht werden $[1,23,25]$. Revisionspflichtige Hämatome werden in $2,6 \%$ bis $5 \%$ der Fälle gesehen, und heilen nach Entlastung problemlos ab [16,24].

Frühe Komplikationen wie oberflächliche aseptische Wundrandnekrosen, Nervenirritationen oder revisionspflichtige Hämatome können durch sorgfältige Präparationstechnik, das Beachten der lokalen Blutversorgung (korrekter Zugang) sowie das Vermeiden jeglichen scharfen Hakenzuges an den Weichteilen minimiert werden.

Tiefe Weichteil- und Knocheninfekte sind gefürchtete Komplikationen, welche in $1,3 \%$ bis $7,0 \%$ nach einer Osteosynthese beobachtet werden $[1,2,18,23]$. Besonders gefährdet sind hierbei offene Frakturen sowie geschlossene Frakturen, welche verspätet ( $>14$ Tage nach dem Unfallereignis) versorgt werden [17,25]. Die Therapie besteht in einem radikalen Débridement, gegebenenfalls der Plättchenentfernung und alternativer Kirschner-Draht-Transfixation mit anschließender Einlage von Antibiotikaträgern und systemischer Antibiotikatherapie. Pseudarthrosen werden mit 0,7 bis $1,6 \%[24,25]$ selten beobachtet. In diesen Fällen ist die 

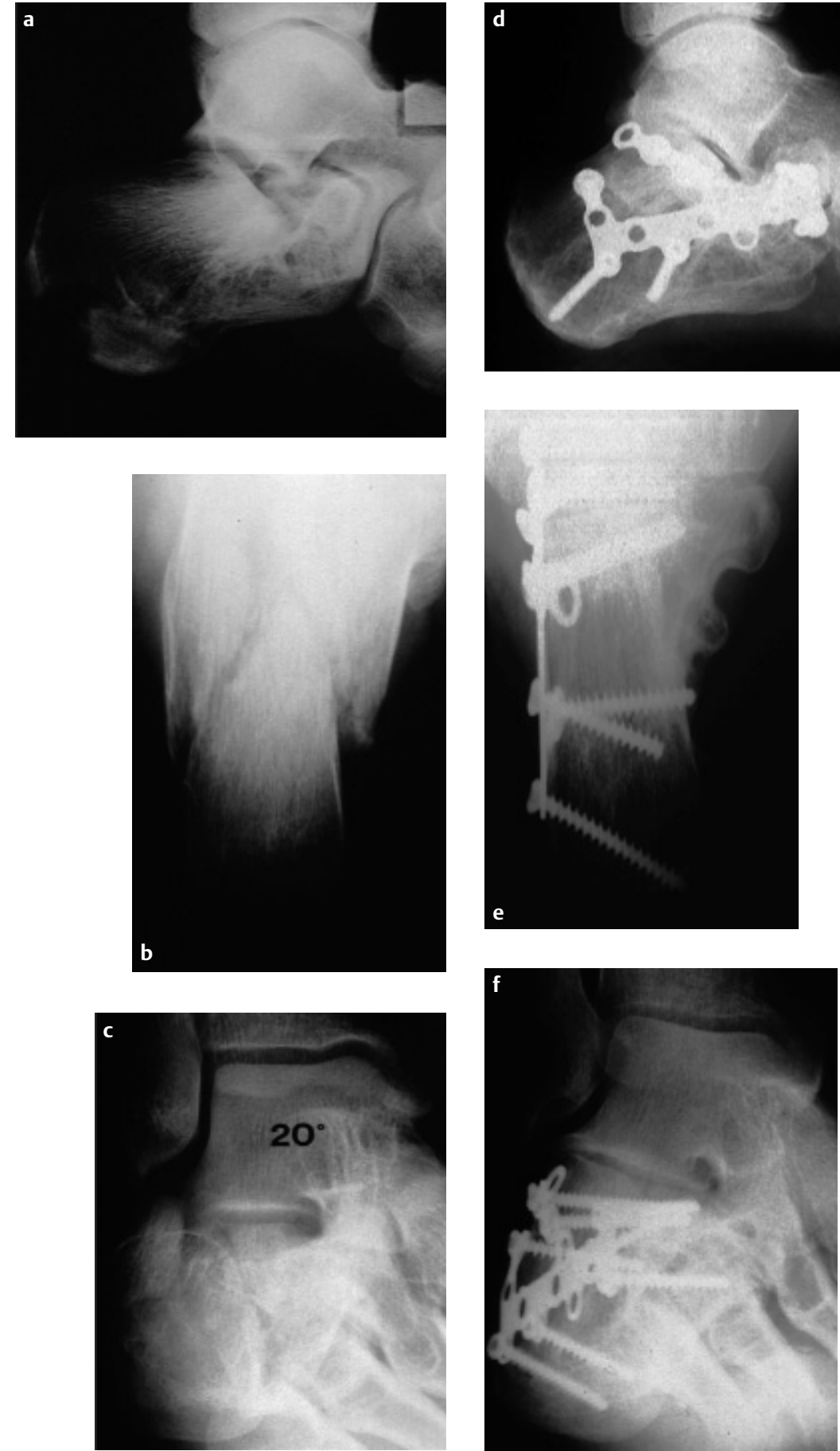

Abb.10 Rekonstruktion einer intraartikulären Calcaneusfraktur. Beachte den aufgehobenen Böhler-Winkel im seitlichen Strahlengang (a), die Verkürzung und Verbreiterung des Fersenbeines in der axialen Aufnahme (b) sowie die Fragmentierung und Depression der posterioren Gelenkfacette in der $20^{\circ}$-BrodenAufnahme (c). Radiologisches Ergebnis nach Osteosynthese mit der AO-Calcaneus-Platte nach Sanders [19], welche an die laterale Fersenbeinwand anmodelliert wird, in denselben Ansichten (d-f).
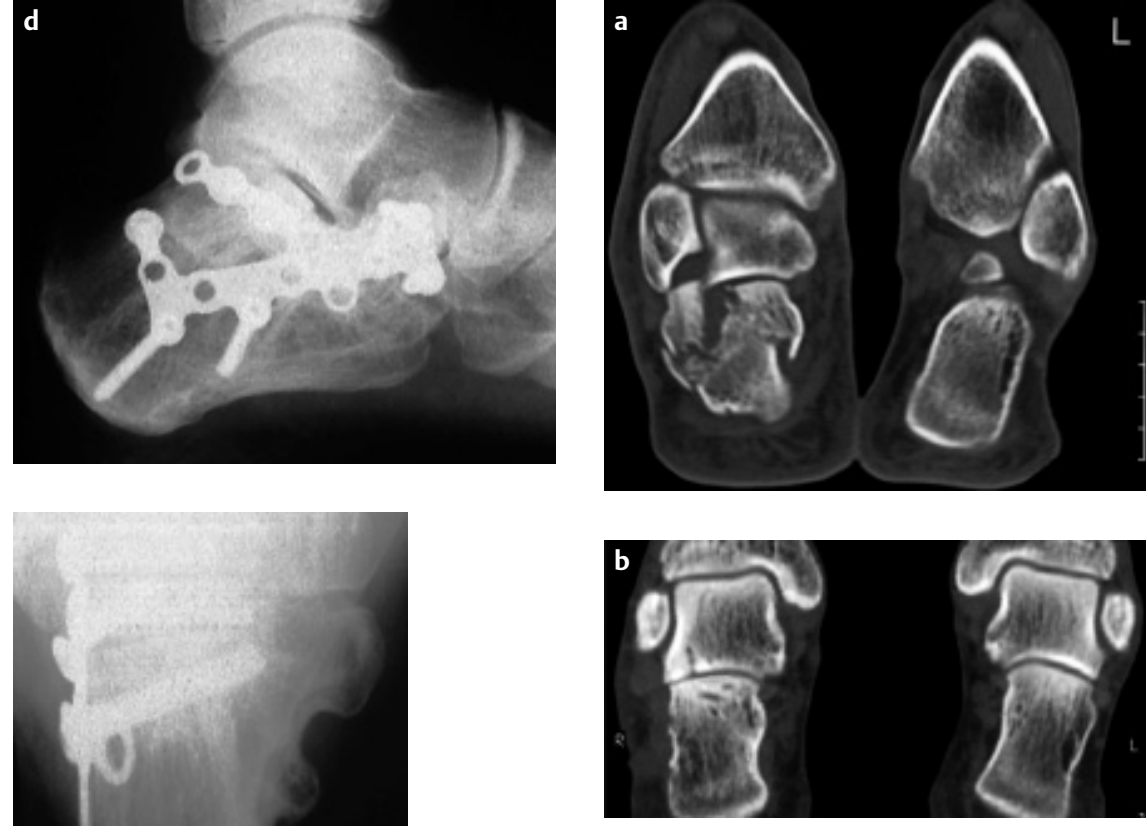

Abb.11 Die CT-Verlaufsdokumentation (selber Patient wie Abb. 10) zeigt die anatomische Rekonstruktion der funktionell entscheidenden posterioren Gelenkfacette nach durchgeführter Implantatentfernung. Die Schraubenkanäle sind unterhalb der Gelenkfläche noch sichtbar.

Revision mit Anfrischung der Fragmentenden, zusätzlicher autologer Knochenplastik und anschließender SchraubenKompressionsosteosynthese angezeigt. Die Komplikationsrate im eigenen Patientengut ist in Tab. 2 dargestellt, sie liegt im Bereich der in größeren Studien angegebenen Zahlen. Auf die besondere Problematik der offenen Calcaneusfrakturen wird mehrfach hingewiesen.

\section{Ergebnisse}

In größeren Serien (über 100 nachuntersuchte Patienten) wurden nach offener Reposition und Plattenosteosynthese in 60 bis $85 \%$ gute und ausgezeichnete Resultate beschrieben $[2,12,18,23]$ wenngleich auch verschiedene Score-Systeme zur Anwendung kamen, was den direkten Vergleich erschwert. Im eigenen Patientengut fanden sich mit dem rein funktionellen modifizierten Merle d'AubignéScore deutlich bessere Resultate, als mit dem \pm 200 -Punkte-Score, welcher zudem strenge morphologische und objektivierbare klinische Kriterien einschließt (Tab.3, Abb.12). Erwartungsgemäß findet sich bei allen Autoren eine negative Korrelation der Ergebnisse mit steigendem Schweregrad der Fraktur und ungenügender Reposition. Dabei wird auf die 
Tab. 2 Komplikationen (Mehrfachnennung möglich) nach operativer Versorgung von Calcaneusfrakturen (eigenes Krankengut 10/1993-12/1999, $\mathrm{n}=275$ )

$\begin{array}{lrr}\text { oberflächliche Wundrandnekrose } & 23 & 6,6 \% \\ \text { revisionspflichtiges Hämatom } & 18 & 5,2 \% \\ \text { Weichteilinfekt* } & 18 & 5,2 \% \\ \text { Knocheninfekt* } & 9 & 2,6 \% \\ \text { verzögerte Knochenheilung } & 1 & 0,3 \% \\ \text { Pseudarthrose } & 1 & 0,3 \%\end{array}$

* Bei 18 von 27 Patienten mit Infektverlauf lag eine II. - III. ${ }^{\circ}$ ig offene Fraktur vor.
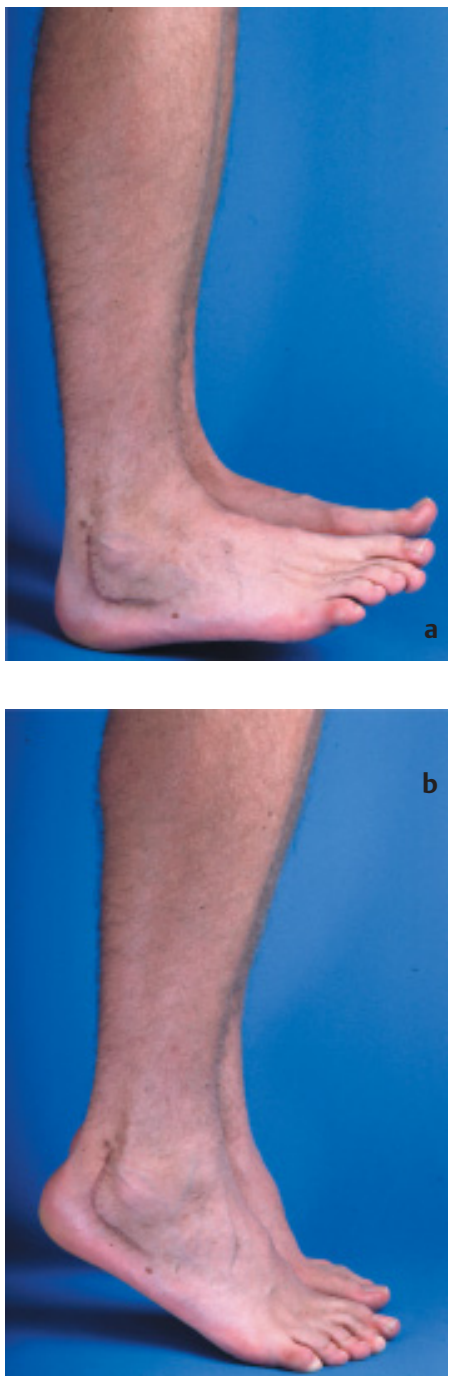

Abb.12 Die klinischen Parameter zur Kontrolle des Behandlungsergebnisses beinhalten u. a. die Beweglichkeit im oberen $(\mathbf{a}, \mathbf{b})$ und unteren Sprungggelenk (c) sowie die Achsenausrichtung des Rückfußes und die plantare Lastverteilung des Fußes im
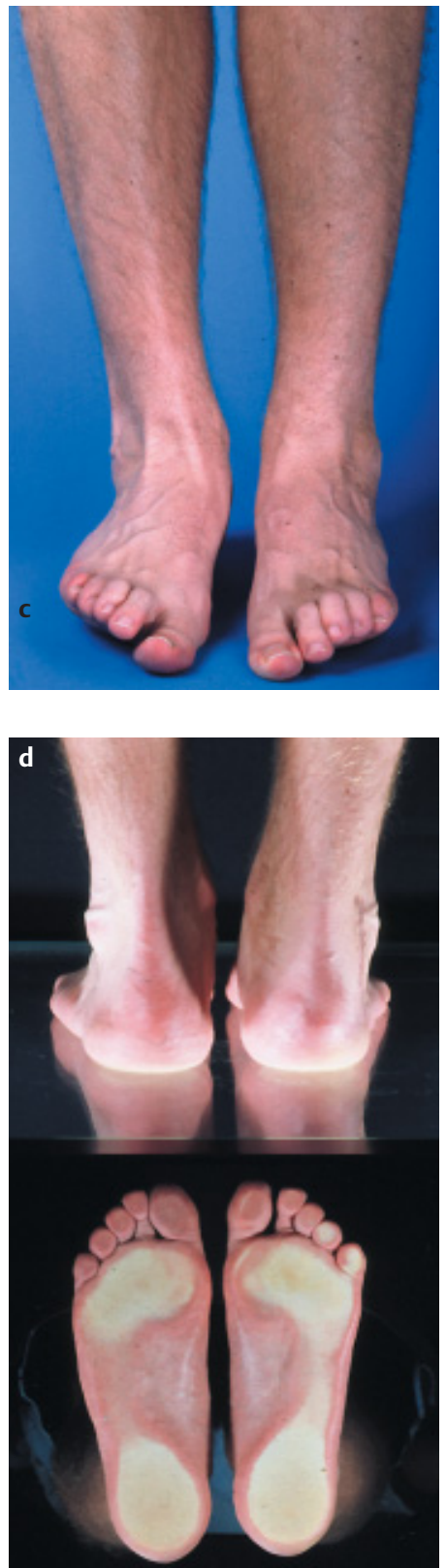
Podogramm (d). Sehr gutes Ergebnis mit dem Merle d'Aubigné- und \pm 200 Punkte-Score (selber Patient wie Abb. 10).
Tab. 3 Behandlungsergebnisse nach chirurgischer Versorgung von Calcaneusfrakturen (eigenes Krankengut, Nachuntersuchungszeitraum 18 Monate postop., $\mathrm{n}=169$ )

\begin{tabular}{lll} 
Score & $\begin{array}{l} \pm 200 \\
\text { Punkte } \\
\text { (Zwipp) }\end{array}$ & $\begin{array}{l}\text { Merle } \\
\text { d'Aubigné } \\
\text { (mod. } \\
\text { n. Buch) }\end{array}$ \\
\hline $\begin{array}{l}\text { sehr gut } \\
\text { gut }\end{array}$ & $10 \%$ & $45 \%$ \\
befriedigend & $57 \%$ & $41 \%$ \\
schlecht & $30 \%$ & $11 \%$ \\
& $3 \%$ & $3 \%$
\end{tabular}

nicht unerhebliche Lernkurve von etwa 2 Jahren bzw. 40 Fällen hingewiesen [19]. Mehrere Autoren haben beobachtet, dass ein schlechteres Resultat insbesondere mit Inkongruenzen im subtalaren Gelenk, weniger mit der Aufrichtung des Böhler-Winkels in den konventionellen Aufnahmen einhergeht. Letzterer hat nur bei deutlichem Korrekturverlust einen negativen Einfluss auf das Behandlungsergebnis [15,16,25].

Während nach der Rehabilitationsstatistik der gewerblichen Berufsgenossenschaften von 1990 die Fersenbeinbrüche noch den höchsten MdE-Index unter allen Frakturen aufwiesen (im Mittel 25.5 v.H.), so beträgt heute nach ersten eigenen Erfahrungen bei 17 abschließend begutachteten berufsgenossenschaftlich versicherten Patienten (II. Rentengutachten) mit isolierten Calcaneusfrakturen die mittlere MdE 18.9 v.H. Die Rate notwendiger sekundärer Arthrodesen konnte von $28 \%$ nach konservativer Therapie (11) im eigenen Vorgehen auf 3.7\% nach operativer Therapie gesenkt werden.

Wenige Studien vergleichen operatives und konservatives Vorgehen bei intraartikulären Calcaneusfrakturen, die einzige prospektiv-randomisierte Untersuchung von Thordarson und Krieger (20) zeigt jedoch eine eindeutige Überlegenheit der Osteosynthese. Verschiedene retrospektive Studien fanden jeweils signifikant bessere Ergebnisse bei operativ versorgten Patienten mit anatomiegerechter Frakturreposition $[13,15,16,18]$.

\section{Schlussfolgerung}

Insgesamt hat das operative Vorgehen mit anatomischer Rekonstruktion und frühfunktioneller Übungsbehandlung zu einer deutlichen Verbesserung der Prognose dieser schweren Verletzungen ge- 
führt. Neuere Tendenzen, wie die arthroskopische Gelenkkontrolle in Verbindung mit offener oder perkutaner Reposition, die frühe Lappendeckung bei offenen Frakturen, der Einsatz winkelstabiler Implantate bei zunehmendem Verzicht auf eine autologe Spongiosaplastik und die Arthrolyse im Rahmen der Implantatentfernung sollten diese Entwicklung zugunsten besserer funktioneller Ergebnisse weiter voranbringen.

\section{Literatur}

${ }^{1}$ Benirschke SK, Sangeorzan BJ. Extensive intraarticular fractures of the foot. Surgical management of calcaneal fractures. Clin Orthop 1993; $128-134$

2 Bèzes H, Massart P, Delvaux D et al. The operative treatment of intraarticular calcaneal fractures. Indications, technique, and results in 257 cases. Clin Orthop 1993; 290: 55-59

${ }^{3}$ Böhler L. Fersenbeinfrakturen. In: Böhler, L.: Die Technik der Knochenbruchbehandlung. Mauderich, Wien 1957, S. 2148-2217

${ }^{4}$ Brenner P, Rammelt S, Gavlik JM, Zwipp H. Die frühe Defektdeckung nach offenen Fußverletzungen. Langenbecks Arch Surg Suppl Kongressbd 1999; 116: 990-992

${ }^{5}$ Buch BD, Myerson MS, Miller SD. Primary subtalar arthrodesis for the treatment of comminuted calcaneal fractures. Foot Ankle Int 1996; 17: 61 - 70

${ }^{6}$ Burdeaux BD. Reduction of calcaneal fractures by the McReynolds medial approach technique and its experimental basis. Clin Orthop 1983; 177: 87-103

${ }^{7}$ Essex-Lopresti P. The mechanism, reduction technique, and results in fractures of the os calcis. Br J Surg 1952; 39: 395-419

${ }^{8}$ Freeman B, Duff S, Allen P et al. The extended lateral approach to the hindfoot. Anatomical basis and surgical implications. J Bone Joint Surg (Br) 1998; 80: 139-142
${ }^{9}$ Gavlik JM, Rammelt S, Zwipp H. Minimal-invasive, arthroskopisch gestützte Osteosynthese von Calcaneusfrakturen. Hefte Unfallchirurg 2000; 282: 172-173

${ }^{10}$ Goff CW. Fresh fractures of the os calcis. Arch Surg 1938; 36: 744-765

${ }^{11}$ James ET, Hunter GA. The dilemma of painful old os calcis fractures. Clin Orthop 1983; 177: $112-115$

${ }^{12}$ Letournel E. Open treatment of acute calcaneal fractures. Clin Orthop 1993; 290: 60 67

${ }^{13}$ Leung KS, Yuen KM, Chan WS. Operative treatment of displaced intra-articular fractures of the calcaneum. Medium-term results. J Bone Joint Surg (Br) 1993; 75: 196-201

14 Omoto H, Sakurada K, Sugi M, Nakamura K. A new method of manual reduction for intra-articular fracture of the calcaneus. Clin Orthop 1983; 177: 104-111

15 Paley D, Hall $H$. Intra-articular fractures of the calcaneus. A critical analysis of results and prognostic factors. J Bone Joint Surg (Am) 1993; 75: 342 - 354

16 Rammelt S, Gavlik JM, Zwipp H. Effizienz der offenen subtalaren Arthroskopie bei de operativen Versorgung intraartikulärer Calcaneusfrakturen. Hefte Unfallchirurg 1997; 268: $99-104$

17 Rammelt S, Gavlik JM, Barthel S et al. Management offener Calcaneusfrakturen. Hefte Unfallchirurg 2000; 282: 29-30

18 Sanders R, Fortin P, DiPasquale A et al. The results of operative treatment of displaced intra-articular calcaneal fractures using a CT scan classification. In: Tscherne $\mathrm{H}$, Schatzker J, Major fractures of the pilon, the talus and the calcaneus. Springer Verlag, Berlin, Heidelberg, New York 1992; 175189.

${ }^{19}$ Sanders R. Intra-articular fractures of the calcaneus. J Bone Joint Surg (Am) 2000; 82: $225-250$

${ }^{20}$ Thordarson DB, Krieger LE. Operative vs. nonoperative treatment of intra-articular fractures of the calcaneus: a prospective randomized trial. Foot Ankle Int 1996; 17: $2-9$
${ }^{21}$ Tornetta P. 3rd. The Essex-Lopresti reduction for calcaneal fractures revisited. J Orthop Trauma 1998; 12: 469-473

${ }^{22}$ Westhues $\mathrm{H}$. Eine neue Behandlungsmethode der Calcaneusfrakturen. Arch Orthop Unfallchir 1934; 35: 121 - 128

${ }^{23}$ Zwipp H, Tscherne H, Thermann H, Weber T. Osteosynthesis of displaced intraarticular fractures of the calcaneus. Results in $123 \mathrm{ca}$ ses. Clin Orthop 1993; 290: 76-86

${ }^{24}$ Zwipp H. Chirurgie des Fußes. Springer Verlag, Wien, New York 1994

${ }^{25}$ Zwipp H, Gavlik JM, Rammelt S. Operative Therapie der Calcaneusfrakturen. In: Moorahrend, U., Funktionelle Behandlung ausgesuchter Verletzungen der unteren Extremität. Sympomed, München 1999; 27 - 37

\section{Dr. med. Stefan Rammelt}

Assistenzarzt

Dr. med. Johann Marian Gavlik

Oberarzt

Sven Barthel

Assistenzarzt

Dr. med. Rene Grass

Oberarzt

Prof. Dr. med. Hans Zwipp

Klinikdirektor

Klinik und Poliklinik für Unfall- und

Wiederherstellungschirurgie

Universitätsklinikum „Carl Gustav

Carus“ der TU Dresden

Fetscherstr. 74

01307 Dresden 